\title{
Modelo para la Inserción de Estudiantes Universitarios de la Universidad Tecnológica de Panamá en Proyectos de Desarrollo de Software
}

\section{Kexy Rodríguez, Nichol Sánchez, and Ramfis Miguelena}

Universidad Tecnológica de Panamá, Panamá, Panamá

\section{Abstract}

The Software Factory of the ICT Research Center is currently implementing a research model that consists in the incorporation of undergraduate students of the Technological University of Panama in software development projects. This model aims to promote the participation of university students on real software development

Corresponding Author: Kexy Rodríguez kexy.rodriguez@utp.ac.pa Received: 15 November 2017 Accepted: 5 January 2018 Published: 4 February 2018

Publishing services provided by Knowledge E

(c) Kexy Rodríguez et al. This article is distributed under the terms of the Creative

Commons Attribution License, which permits unrestricted use and redistribution provided that the original author and source are credited.

Selection and Peer-review under the responsibility of the ESTEC Conference Committee.

G OPEN ACCESS projects, and to build a research culture. The model consists of five phases: Project planning, selection of students, provide a 45 -hour training to selected students on ICT Research Center development guidelines, assign and insert students in software projects (they will also be assigned to a mentor), and give the opportunity to high performance students to be recruited byan ICT Research group. It should be noted that student participation is voluntary, so they do not receive final compensation.

Keywords: Student's insertion, volunteering, software development

\section{Resumen}

La Unidad de Fábrica de Software del Centro de Investigación, Desarrollo e Innovación en Tecnologías de la Información y las Comunicaciones está implementando actualmente un modelo de investigación que consiste en la Inserción de estudiantes universitarios de la Universidad Tecnológica de Panamá en proyectos de desarrollo de software. Este proyecto tiene como objetivo promover la participación de los estudiantes universitarios en proyectos reales de desarrollo de software y a la vez inculcarle una cultura investigativa. El modelo se compone de cinco fases: iniciando con una planificación del proyecto, seguido por un proceso de selección de estudiantes, inducción de 45 horas de capacitación a los estudiantes seleccionados según las directrices de CIDITIC, asignación e incorporación de los estudiantes en un proyecto de software (serán asignados a un mentor) y la oportunidad de los estudiantes sobresalientes de pertenecer a un grupo de investigación de CIDITIC. Cabe destacar 
que la participación del estudiante es de forma voluntaria, por lo que no recibe remuneración económica.

Palabras claves: Inserción estudiantil, voluntariado, desarrollo de software

\section{Introducción}

El modelo de inserción estudiantil tiene el propósito de brindar a los estudiantes del área de computación una experiencia profesional en proyectos de desarrollo de software en un Centro de Investigación Universitario. Este modelo está siendo implementado por la Unidad de Fábrica de Software del Centro de Investigación, Desarrollo e Innovación en Tecnologías de la Información y las Comunicaciones - CIDITIC de la Universidad Tecnológica de Panamá - UTP. La UTP cuenta con 5 centros de investigación, cada uno de ellos especializado en áreas específicas de investigación, tales como agroindustria, energía, ambiente, infraestructura, logística, transporte, manufactura, ciencia de los materiales, tecnologías de la información y comunicaciones, entre otros (UTP, 2012). La Unidad de Fábrica de Software de CIDITIC apoya con el desarrollo de software a proyectos de investigación; además de automatizar procesos a los centros de investigación de la UTP que lo soliciten. Dentro de los tipos de servicios específicos que ofrece la Fábrica de Software se puede mencionar: aplicaciones web, sistemas de información, base de datos, asesoría y consultoría, por lo que se está aprovechando para que los estudiantes tengan la oportunidad de incorporarse en algún proyecto donde puedan obtener un aporte significativo en su desarrollo académico y profesional.

Es evidente que la falta de experiencia profesional es una dificultad que presentan los jóvenes para encontrar su primer empleo una vez terminado la universidad. Según un estudio presentado por Manpower Group en mayo de 2014, reflejó que el 50\% de los jóvenes panameños siente que son discriminado al momento de conseguir un empleo por no tener experiencia profesional (Chandiramani, 2014). A nivel regional, un informe de la Comisión Económica para América Latina y el Caribe - CEPAL, presentado a las Naciones Unidas en el 2006, mostró que los egresados universitarios tenían ciertos privilegios al momento de conseguir su primer empleo; sin embargo, los nuevos profesionales encontraban una gran dificultad de insertarse al mercado laboral por no tener experiencia debido a que en su desarrollo académico no contenía el factor práctico que requería el mercado (CEPAL, 2006). A nivel mundial, según la Oficina 
Mundial del Trabajo, la tasa de desempleo juvenil es alta y uno de los factores que incide, es la carencia de competencias y experiencia (OIT, 2012). Esto demuestra que la falta de experiencia profesional es un obstáculo que presentan muchos graduados al momento de conseguir su primer empleo con un salario remunerado. Por otro lado, se prevé que en Panamá el porcentaje de nuevas contrataciones para el 2017 es del 8\% según la Encuesta de Expectativas de Empleo ManpowerGroup (ManpowerGroup, 2017), sumando a esto, los grandes cambios en el sector económico de la Tecnología y Comunicación en Panamá, ha evolucionado en los últimos años dando como resultado que la industria necesite profesionales más competitivos (Rios, 2014), obligando a los estudiantes universitarios obtener una experiencia profesional antes de culminar sus estudios superiores para competir en el mercado.

Teniendo estos datos como evidencia, la Fábrica de Software con el fin de aportar a la calidad de la enseñanza e investigación diseñó un modelo para la inserción de Estudiantes universitarios a proyectos reales de software por medio del voluntariado, donde los estudiantes pasan por un proceso de selección, capacitación, inserción en un proyecto real de software y participación de un grupo de investigación en el Centro CIDITIC.

El presente artículo detalla cómo está estructurado el Modelo para la Inserción de Estudiantes Universitarios de la Universidad Tecnológica de Panamá en proyectos de desarrollo de software, el mismo está organizado de la siguiente manera: metodología, resultados y conclusión.

\section{Metodología}

El diseño curricular de la Universidad Tecnológica de Panamá está basado en estudios que indican las necesidades del mercado laboral panameño, donde la relación directa del aprendizaje mediante la práctica es uno de los aspectos más relevantes de esta estructura curricular (UTP, 2013). En este sentido el desarrollo de la metodología se consideró el enfoque del diseño curricular de la UTP siguiendo la misma finalidad, debido a que la participación de estudiantes universitarios de computación en proyectos reales de desarrollo de software fortalece las actividades cognoscitiva del estudiante universitario, por lo que este proyecto tiene los mecanismos necesarios para que los estudiantes tengan la experiencia teórica práctica en la universidad sin necesidad de movilizarse a otro lugar.

La metodología del proyecto de inserción estudiantil por medio del voluntariado está conformado por cinco importantes fases: planeación del modelo de inserción 
estudiantil, selección de estudiantes, inducción de los estudiantes, inserción de los estudiantes a un proyecto de software e inserción de los estudiantes a un grupos de investigación de CIDITIC.

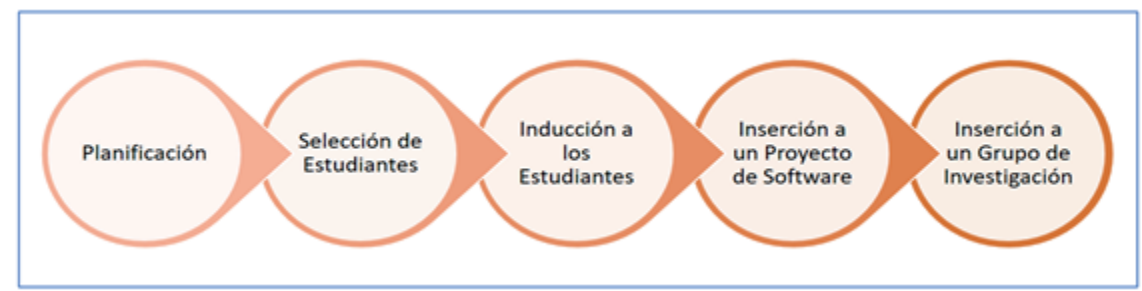

Figura 1: Modelo de Inserción de Estudiantes Universitarios en Proyectos de Desarrollo de Software.

Cabe destacar que para completar todas las fases toma alrededor de un año desde que el estudiantes es seleccionado. Este modelo está siendo implementado desde agosto del 2016.

\subsection{Planificación}

El enfoque de esta primera fase fue la elaboración de una planificación estratégica por objetivos. Este método de planificación permite incorporar a todos los involucrados desde el inicio del proyecto, enfocándose en el cumplimiento de las metas y las acciones a tomar para cualquier eventualidad (Trigoso, 2014). Al establecerse una planificación realista, se promovió la colaboración y compromiso de los participantes en el desarrollo del proyecto.

Como primer paso se identificó al recurso humano que conformaría el equipo para llevar a cabo el proyecto; este equipo fue conformado por investigadores, docentes, desarrolladores de software y gestor de proyectos. Con el equipo de trabajo se estableció el alcance del proyecto, el objetivo general y los específicos que se deseaban cumplir. De igual forma dentro de la planificación se desarrollaron sub-fases, las cuales fueron: revisión bibliográfica y antecedentes, establecimiento de hipótesis, elaboración de un procedimiento de selección de estudiantes, elaboración de un plan de capacitación, identificación de proyectos de desarrollo de software que serán utilizado en el proyecto, elaboración de estrategias de divulgación de resultados, elaboración de cronograma y presupuesto. Todas estas sub-fases fueron incorporadas en un documento escrito como una propuesta de proyecto, el cual se presentó y fue aprobado por la dirección del Centro de Investigación, Desarrollo e Innovación en Tecnologías de la Información y las Comunicaciones - CIDITIC. 
Cabe destacar que el documento debe ser actualizado cada vez que se culmine la implementación del modelo de inserción estudiantil con el fin de mejorar la planificación y las estrategias del proyecto.

\subsection{Selección de Estudiantes}

El proceso de selección es un factor importante para las organizaciones al momento de requerir un personal para un puesto particular. Para el éxito del proceso de selección, los objetivos y funciones deben estar previamente definidos (Alonso, Moscoso, \& Dámaris, 2015). Рara el caso del modelo de inserción estudiantil de la UTP, el perfil del estudiante se basó en las competencias de un desarrollador de aplicaciones de la Unidad de Fábrica de Software de CIDITIC, debido a que los estudiantes serían incorporados en proyectos reales de desarrollo de software. Sin embargo, a los estudiantes no se les podría exigir un alto nivel de conocimiento, ya que ellos están en su desarrollo académico; pero, si es necesario identificar con qué conocimiento cuentan hasta el momento en cuanto a análisis, diseño, base de datos y programación de software.

Para la puesta en marcha del proyecto, se elaboró un plan estratégico para la convocatoria de estudiantes interesados. Este plan consistía en atraer estudiantes a través de una red de conocidos. Se requirió la recomendación de un solo estudiante por parte de un docente, de la Facultad de Ingeniería de Sistemas Computacionales de la UTP, para que este se convirtiera en un agente multiplicador motivando a otros estudiantes y así sucesivamente armar la red de conocidos. La estrategia es muy parecida a la red de mercadeo de multinivel. La red de mercadeo de multinivel se utiliza para vender u ofrecer un producto directamente a otros individuos convirtiendo en consumidores y estos individuos a su vez buscan a otros consumidores para finalmente armar una red de mercado (Medina, 2015). Aunque, en este proyecto no se estaba promocionando ni vendiendo un producto, sino que se está ofreciendo la oportunidad de pertenecer a un programa de voluntariado, donde ellos obtendrían experiencia profesional en desarrollo de software.

Aplicando esta estrategia, 14 estudiantes de la Facultad de Ingeniería de Sistemas Computacionales se interesaron en ingresar en el programa de voluntariado, como se refleja en la Tabla 1.

Рara la fase de selección, se elaboró una estructura de entrevista personalizada y una prueba diagnóstica. La entrevista era para conocer los intereses y aspiraciones personales en cuanto desarrollo profesional; ésta era realizada por el coordinador de la Fábrica de Software y un Investigador en el área de Ingeniería de Software. La prueba 
TABLA 1: Cantidad de estudiantes interesados por año académico.

Año Académico
I
II
III
IV
Total

\begin{tabular}{|c|} 
Cantidad \\
1 \\
10 \\
12 \\
1 \\
14
\end{tabular}

diagnóstica fue elaborada por el equipo de la Fábrica de Software y la misma consistía en un problema a resolver, donde se evaluaban los conocimientos generales en cuanto a análisis de sistemas, diseño de base datos y programación de software.

TABLA 2: Puntaje promedio obtenido de los estudiantes de la prueba diagnostica.

\begin{tabular}{c} 
Sección \\
\hline Análisis de Sistemas \\
\hline Diseño de base de datos \\
\hline Programación \\
Total
\end{tabular}

Puntaje promedio obtenido por los
estudiantes
$60 \%$
$40 \%$
$30 \%$
$43 \%$

La prueba diagnóstica tenía un puntaje total de 100; sin embargo, el resultado de la prueba no definía la selección del estudiante, ya que se valoró el entusiasmo e interés del estudiante de aprender. Como se puede apreciar en la tabla 2 existía una deficiencia general en los conocimientos en cuando a desarrollo de software. Este resultado fue muy importante para el equipo del proyecto puesto que con esto se mejoró el plan de capacitación desarrollado en la fase de planificación.

Además de la entrevista y la prueba diagnóstica, los estudiantes tenían que presentar documentación certificada por la universidad: su índice académico y certificación que eran estudiantes regulares matriculados. De los 14 estudiantes interesados se seleccionaron 9 para ingresar al programa de voluntariado el cual representó el $64 \%$. En la selección de los estudiantes participaron todos los integrantes del proyecto del modelo para la inserción de estudiantes universitarios de la Universidad Tecnológica de Panamá en proyectos de desarrollo de software. 


\subsection{Inducción a los Estudiantes}

Este criterio fue considerado en la planificación, pero fue mejorado una vez obtenido los resultados de la prueba diagnóstica de los estudiantes. Para esto se elaboró un plan de capacitación de 45 horas el cual estaba conformado por 9 cursos que se encuentran enfocados a la metodología de trabajo de la Fábrica de Software y una actividad para reforzar el trabajo en equipo. Los temas de los cursos fueron: Análisis de Requerimientos, Diseño de Prototipos, Diseño de Base de Datos, Front-end Framework Bootstrap y Javascript; Lenguaje de Programación PHP, Framework Laravel, Jquery Table, Validación con Jquery y Desarrollo para Móvil. En la capacitación se aplicó mecánicas de gamificación para dinamizar las clases. La gamificación es una estrategia que utiliza mecánica de juegos para fomentar una conducta o compromiso en las personas en una forma divertida (Ramírez, 2014).

Los estudiantes seleccionados se comprometieron a asistir a la capacitación de 45 horas y al voluntariado mediante la firma de un acuerdo entre el estudiante y el Centro de Investigación CIDITIC. La capacitación tenía un valor de \$750.00 pero a los estudiantes se les otorgó la beca completa siempre y cuando cumplieran con el acuerdo firmado.

\subsection{Inserción a un Proyecto de Software}

Una vez culminada la capacitación sobre desarrollo de software, a cada estudiante se le asigna un mentor que lo asesore en las actividades asignadas. Los mentores son especialistas en desarrollo de software que laboran en la Unidad de Fábrica de Software del Centro de Investigación CIDITIC.

En cuanto a los proyectos que fueron asignados los estudiantes, todos son proyectos reales que coordina y desarrolla la Unidad de Fábrica de Software, de los cuales se puede mencionar proyectos tales como: sitios web, sistemas de información, sistemas de inscripción online, sistemas de reportes y aplicaciones móviles. Los estudiantes participan en los proyectos que sus mentores coordinan o desarrollan. Adicionalmente, se le hace entrega de una notificación por escrito de las actividades, cronograma y entregables que deberán seguir.

En cuanto a disponibilidad, las actividades asignadas por el mentor son acorde a la disponibilidad del horario de clases del estudiante. Se requiere que mínimo una vez a la semana el estudiante se reúna con su mentor. Cabe destacar que durante el 
voluntariado a los estudiantes se les motiva y se les guía para la escritura de artículos científicos.

\subsection{Inserción a un Grupo de Investigación}

El Centro de Investigación, Desarrollo e Innovación en Tecnologías de la Información y las Comunicaciones - CIDITIC cuenta con 6 grupos de investigación, los cuales son: Digital Media, Ingeniería Ontológica y Web Semántica; Usabilidad y Experiencia de Usuario; Gamificación, Salud Electrónica y Seguridad en las TIC (UTP, 2017). La estrategia que se ha utilizado para incluir a los estudiantes en proyectos de investigación ha sido que, durante la participación como voluntario, los grupos de investigación realicen charlas y conferencias sobre los proyectos que están desarrollando. Seguido los estudiantes deben decidir a qué grupo desean pertenecer de acuerdo a sus intereses investigativas; una vez el estudiante termine con el voluntariado, puede incorporarse a un grupo de investigación y tener la oportunidad de ser contratado como asistente de investigación estudiantil.

\section{Resultados}

La implementación del modelo ha sido muy positiva ya que los estudiantes voluntarios han reducido la brecha entre los conocimientos académicos, prácticos e investigativos. Los resultados de este proyecto se han divido en varios aspectos:

\subsection{Académicos}

El conocimiento de los estudiantes en cuanto al desarrollo de software antes y después de la capacitación aumentó considerablemente. Al final de cada curso se le aplicaba una prueba del tema dictado y al finalizar la capacitación una evaluación final. La prueba final abarcaba todos los temas dados en la capacitación y de la prueba diagnóstica, donde el puntaje promedio fue de un 80/100. Si comparamos este resultado con el resultado promedio obtenido en la prueba diagnóstica, los estudiantes obtuvieron un incremento del $\mathbf{8 6 \%}$ en cuando a conocimiento sobre desarrollo de software.

\footnotetext{
$\%$ de creciemiento

$=\frac{\text { Resultado promedio obtenido en la prueba final }- \text { Resultado promedio obtenido en la prueba diagnstica }}{}$ Resultado promedio obtenido en la prueba diagnstica
}

$\%$ de creciemiento $=\frac{80-43}{43}=0.86 \times 100=86 \%$ 
Al finalizar la capacitación a los estudiantes se les entregó un certificado expedido por la Universidad Tecnológica de Panamá de 45 horas.

\subsection{Profesional}

Los estudiantes del programa de voluntariado han tenido una experiencia el cual ha ayudado en su crecimiento profesional. Su aporte ha sido significativo en los proyectos de software que están participando con sus mentores como indica la lista de proyectos de la Tabla 3. Igualmente, los mentores han ganado experiencia en cuanto a coordinación y supervisión de personal.

En promedio, cada voluntario ha tenido un aporte semanal de 10 horas durante 4 meses.

TABLA 3: Listado de proyectos donde se insertaron a los estudiantes.

\# Proyecto
$1 \begin{aligned} & \text { Automatización del Laboratorio de Ensayo de } \\ & \text { Materiales del Centro de Experimental de Ingeniería } \\ & \text { de la UTP }\end{aligned}$
2 Sistema de Gestión Documental
$3 \begin{aligned} & \text { Sistema de Inscripción de la Jornada de Iniciación } \\ & \text { Científica }\end{aligned}$
$4 \begin{aligned} & \text { Sistema de Educación Continua } \\ & \text { Sitio Web y Sistema de Inscripción del Congreso de } \\ & \text { Internacional de Ingeniería Mecánica de la UTP }\end{aligned}$

\begin{tabular}{|l|c|}
\hline Fase del Proyecto & $\begin{array}{c}\text { Cantidad de } \\
\text { voluntarios }\end{array}$ \\
\hline $\begin{array}{l}\text { Desarrollo de } \\
\text { Reportes }\end{array}$ & 1 \\
\hline $\begin{array}{l}\text { Análisis de } \\
\text { Requerimiento }\end{array}$ & 2 \\
\hline $\begin{array}{l}\text { Análisis, diseño y } \\
\text { Desarrollo }\end{array}$ & 1 \\
\hline $\begin{array}{l}\text { Reingeniería e } \\
\text { implementación }\end{array}$ & 1 \\
\hline $\begin{array}{l}\text { Análisis, diseño y } \\
\text { Desarrollo }\end{array}$ & 2 \\
\hline
\end{tabular}

\subsection{Investigación}

En cuanto a investigación, a los estudiantes se les motiva a participar en actividades de investigación por medio de charlas y conferencia de los grupos de investigación de CIDITIC. De los 9 estudiantes seleccionados 2 han sido contratados como ayudante de investigación estudiantil, el cual se han incorporado en proyectos colaborando directamente con investigadores y uno de ellos ya hizo una publicación científica. Los otros 7 estudiantes que están en sus labores de voluntariado escribirán un artículo apoyado con su mentor sobre la experiencia obtenida en el proyecto asignado o sobre algún tema de actualidad. Por último, se espera que los estudiantes voluntarios se incorporen a un grupo de investigación. 


\section{Conclusión}

La implementación del modelo para la inserción de estudiantes universitarios ha permitido agilizar el desarrollo de los proyectos gestionados en la Unidad de Fábrica de Software del Centro de Investigación CIDITIC; incluso a dar respuesta a otros proyectos que estaban pendiente por desarrollar.

Sin embargo, es necesario indicar que la aplicación del modelo ha incrementado las labores administrativas en la Unidad de Fábrica de Software, debido a que los desarrolladores de software han tomado el rol de mentores, en donde cada uno de ellos debe dar seguimiento a los estudiantes voluntarios y continuar con el desarrollo de sus proyectos previamente asignados.

En relación a los estudiantes universitarios, cabe destacar que los mismos han tenido un desarrollo teórico práctico en cuanto al conocimiento obtenido durante el voluntariado. La experiencia teórica práctica ganada en un ambiente laboral permitirá tener un impacto significativo tanto en su carrera universitaria como en el mercado laboral. Adicionalmente, se les ha promovido la cultura investigativa que es un factor importante en el desarrollo académico universitario.

Finalmente, рага el éxito de la implementación del modelo es importante la constante actualización y mejoras de las estrategias, basada en las experiencias, para fortalecer el modelo en las próximas implementaciones.

\section{Referencias}

[1] Alonso, P., Moscoso, S., \& Dámaris, C. (2015). Procedimientos de selección de personal en pequenas y medianas empresas españolas. Journal of Work and Organizational Psychology, 31, 79-89.

[2] CEPAL. (2006). Los Jóvenes y el Empleo en América Latina Desafíos y Perspectivas ante el Nuevo Escenario Laboral. Comisión Económica para América Latina y el Caribe - CEPAL. Bogotá: Mayol Ediciones S.A. Retrieved from www. eclac.cl

[3] Chandiramani, R. (2014, mayo 21). Falta de experiencia limita a los jóvenes a conseguir empleo. La Estrella de Panamá.

[4] ManpowerGroup. (2017). Encuesta de Expectativas de Empleo ManpowerGroup Panamá. Panamá: ManpowerGroup Panamá. Retrieved from wWw.manpowergroup.com.mx

[5] Medina, J. (2015, Noviembre). Sistema de Mercadeo Multinivel. Retrieved from Gimnasio Moderno NET: http://www.gimnasiomoderno.net/aula/pluginfile. 
$\mathrm{php} / 7436 /$ mod_folder/content/0/Sistema $\% 20$ de $\% 20$ mercadeo $\% 20$ multinivel.

pdf ? f orcedownload $=1$

[6] OIT. (2012). Impulso a la Empleabilidad de los Jóvenes Desfavorecidos. Ginebra, Suiza: International Labour Organization.

[7] Ramírez, J. (2014). Gamificación: Mecánica de juegos en tu vida personal y profesional. Madrid: Sclibro.

[8] Rios, B. (2014, abril 8). ¿Escasez de talento en Panamá? Revista IT Now. Retrieved from http://revistaitnow.com/escasez-de-talento-en-panama-2/.

[9] Trigoso, A. (2014). Planeación de proyectos orientada a objetivos - método ZOPP. Actualidad Gubernamental № 64, 4-6.

[10] UTP. (2012). Plan de Desarrollo Institucional (PDI) 2013-2017. Panamá, Panamá: Universidad Tecnológica de Panamá.

[11] UTP. (2013). Modelo Educativo de la Universidad Tecnológica de Panamá. Universidad Tecnológica de Panamá, Panamá.

[12] UTP. (2017, 5 22). Listado de Grupos de Investigación. (Universidad Tecnológica de Panamá) Retrieved from Universidad Tecnológica de Panamá: http://www.utp.ac.pa/listado-de-grupos-de-investigacion

\section{Authorization and Disclaimer}

Authors authorize ESTEC to publish the paper in the conference proceedings. Neither ESTEC nor the editors are responsible either for the content or for the implications of what is expressed in the paper. 